CREAT. MATH. INFORM.

Volume 25 (2016), No. 2,

Pages 197 - 203
Online version at https : //creative-mathematics. cunbm . utcluj. ro/

Print Edition: ISSN 1584 - 286X; Online Edition: ISSN 1843 - 441X

DOI: https:/ /doi.org/10.37193/CMI.2016.02.11

\title{
Rate of growth of polynomials with restricted zeros
}

\author{
AbDullah Mir and Q. M. DAWOOD
}

ABSTRACT. In this paper we consider for a fixed $\mu$, the class of polynomials $P(z)=a_{0}+\sum_{\nu=\mu}^{n} a_{\nu} z_{\nu}, 1 \leq$ $\mu \leq n$, of degree at most $n$ not vanishing in the disk $|z|<k, k>0$. For any $\rho>\sigma \geq 1$ and $0<r \leq$ $R \leq k$, we investigate the dependence of $\|P(\rho z)-P(\sigma z)\|_{R}$ on $\|P\|_{r}$ and derive various refinements and generalizations of some well known results.

\section{INTRODUCTION}

Let $P_{n}$ be the class of polynomials $P(z)=\sum_{\nu=0}^{n} a_{\nu} z_{\nu}$ of degree at most $n$. For $P \in P_{n}$, we define

$$
\begin{gathered}
\|P\|:=\max _{|z|=1}|P(z)|,\|P\|_{R}:=\max _{|z|=R}|P(z)|, \\
\|P(\rho z)-P(\sigma z)\|_{R}:=\max _{|z|=R}|P(\rho z)-P(\sigma z)| \\
\text { and } m:=\min _{|z|=k}|P(z)| .
\end{gathered}
$$

If $P \in P_{n}$, then concerning the estimate of the maximum of $\left|P^{\prime}(z)\right|$ on the unit circle $|z|=1$ and the estimate of the maximum of $|P(z)|$ on a larger circle $|z|=R>1$, we have

$$
\left\|P^{\prime}\right\| \leq n\|P\|
$$

and

$$
\|P\|_{R} \leq R^{n}\|P\| \text {. }
$$

Inequality (1.1) is a well-known result of S. Bernstein (for reference see [15, p-508]), whereas inequality (1.2) is a simple deduction from maximum modulus principle (see [15, p-405]).

If we restrict ourselves to the class of polynomials $P \in P_{n}$ with $P(z) \neq 0$ in $|z|<1$, then Erdös conjectured and later Lax (for reference see [15, p-562]), verified that the inequality (1.1) can be replaced by

$$
\left\|P^{\prime}\right\| \leq \frac{n}{2}\|P\| \text {. }
$$

As an extension of (1.3), it was shown by Malik (for reference see [15, p-563]), that if $P \in P_{n}$ and $P(z) \neq 0$ in $|z|<k, k \geq 1$, then

$$
\left\|P^{\prime}\right\| \leq \frac{n}{1+k}\|P\| \text {. }
$$

Received: 14.01.2016. In revised form: 30.03.2016. Accepted: 08.04.2016 2010 Mathematics Subject Classification. 30A10, 30C10, 30C15.

Key words and phrases. Polynomials, inequalities, maximum modulus principle, Growth.

Corresponding author: Abdullah Mir; mabdullah_mir@yahoo.co.in 
Bidkham and Dewan [3] obtained a generalization of inequality (1.4) and proved that if $P \in P_{n}$ and $P(z) \neq 0$ in $|z|<k, k \geq 1$, then

$$
\left\|P^{\prime}\right\|_{r} \leq \frac{n(r+k)^{n-1}}{(1+k)^{n}}\|P\|
$$

where $1 \leq r \leq k$.

In the literature, there already exist various refinements and generalizations of (1.3), (1.4) and (1.5), for example see Mir, Dewan and Singh [10]-[11], Dewan, Singh and Mir [5], Mir, Dewan, Singh and Dar [13], Mir and Dar [12], Govil and Nyuydinkong [9], Gardner, Govil and Weems [6]-[7],Gardner, Govil and Musukula[8],etc.

In this paper, we denote by $P_{n, \mu}, 1 \leq \mu \leq n$, the linear space of all polynomials of the form $P(z)=a_{0}+\sum_{\nu=\mu}^{n} a_{\nu} z_{\nu}$ of degree at most $n$. Note that $P_{n, 1}=P_{n}$. Aziz and Shah [2] improved as well as extended the inequalities (1.3), (1.4) and (1.5) by showing that if $P \in P_{n, \mu}$ and $P(z) \neq 0$ in $|z|<k, k>0$, then for $0<r \leq R \leq k$,

$$
\left\|P^{\prime}\right\|_{R} \leq \frac{n R^{\mu-1}\left(R^{\mu}+k^{\mu}\right)^{\frac{n}{\mu}-1}}{\left(k^{\mu}+r^{\mu}\right)^{\frac{n}{\mu}}}\left(\|P\|_{r}-m\right) .
$$

More recently Aziz and Aliya [1] besides proving some other results, also calculated the growth of $\|P(\rho z)-P(z)\|_{R}$ where $\rho>1,0<r \leq R \leq k$ and proved the following interesting generalization of inequality (1.6).

Theorem 1.1. If $P \in P_{n, \mu}$ and $P(z) \neq 0$ in $|z|<k, k>0$, then for every $\rho>1$ and $0<r \leq R \leq k$,

$$
\|P(\rho z)-P(z)\|_{R} \leq \frac{R^{\mu}\left(\rho^{n}-1\right)}{r^{\mu}+k^{\mu}}\left(\frac{R^{\mu}+k^{\mu}}{r^{\mu}+k^{\mu}}\right)^{\frac{n}{\mu}-1}\left(\|P\|_{r}-m\right) .
$$

Note 1: If we divide both sides of (1.7) by $\rho-1$ and let $\rho \rightarrow 1$, we get (1.6). As a refinement of Theorem (1.1), Mir and Dar [12] proved the following result by involving some of the coefficients of the polynomial $P(z)$.

Theorem 1.2. If $P \in P_{n, \mu}$ and $P(z) \neq 0$ in $|z|<k, k>0$, then for every $\rho>1,0<r \leq R \leq k$ and $0 \leq \lambda \leq 1$,

$$
\begin{aligned}
& \|P(\rho z)-P(z)\|_{R} \leq\left(\rho^{n}-1\right)\left(\frac{\left(\frac{\rho^{\mu}-1}{\rho^{n}-1}\right) \frac{\left|a_{\mu}\right|}{\left|a_{0}\right|-\lambda m} k^{\mu+1} R^{\mu}+R^{\mu+1}}{R^{\mu+1}+k^{\mu+1}+\left(\frac{\rho^{\mu}-1}{\rho^{n}-1}\right) \frac{\left|a_{\mu}\right|}{\left|a_{0}\right|-\lambda m}\left(k^{\mu+1} R^{\mu}+k^{2 \mu} R\right)}\right) \\
& \times \exp \left\{n \int_{r}^{R} \frac{\frac{\mu}{n} \frac{\left|a_{\mu}\right|}{\left|a_{0}\right|-\lambda m} k^{\mu+1} \varsigma^{\mu-1}+\varsigma^{\mu}}{\varsigma^{\mu+1}+\frac{\mu}{n} \frac{\left|a_{\mu}\right|}{\left|a_{0}\right|-\lambda m}\left(k^{\mu+1} \varsigma^{\mu}+k^{2 \mu} \varsigma\right)+k^{\mu+1}} d \varsigma\right\}\left(\|P\|_{r}-\lambda m\right) .
\end{aligned}
$$

Note 2: If we divide both sides of (1.8) by $\rho-1$, let $\rho \rightarrow 1$ and take $\lambda=1$, we get a result of Chanam and Dewan [4, Theorem (2.4)].

\section{MAIN RESULTS}

In this paper, we shall prove the following result which generalises and refines the bounds of Theorems (1.1) and (1.2). More precisely, we prove 
Theorem 2.3. If $P \in P_{n, \mu}$ and $P(z) \neq 0$ in $|z|<k, k>0$, then for every $\rho>\sigma \geq 1,0<r \leq$ $R \leq k, 0 \leq \lambda \leq 1$ and $n>2$, we have,

$$
\begin{aligned}
& \|P(\rho z)-P(\sigma z)\|_{R} \leq\left(\frac{\left(\frac{\rho^{\mu}-\sigma^{\mu}}{\rho^{n}-\sigma^{n}}\right) \frac{\left|a_{\mu}\right|}{\left|a_{0}\right|-\lambda_{m}} k^{\mu+1} R^{\mu}+R^{\mu+1}}{R^{\mu+1}+k^{\mu+1}+\left(\frac{\rho^{\mu}-\sigma^{\mu}}{\rho^{n}-\sigma^{n}}\right) \frac{\left|a_{\mu}\right|}{\left|a_{0}\right|-\lambda m}\left(k^{\mu+1} R^{\mu}+k^{2 \mu} R\right)}\right) \\
& \times\left[\left(\rho^{n}-\sigma^{n}\right) \exp \left\{n \int_{r}^{R} \frac{\frac{\mu}{n} \frac{\left|a_{\mu}\right|}{\left|a_{0}\right|-\lambda m} k^{\mu+1} \varsigma^{\mu-1}+\varsigma^{\mu}}{\varsigma^{\mu+1}+\frac{\mu}{n} \frac{\left|a_{\mu}\right|}{\left|a_{0}\right|-\lambda m}\left(k^{\mu+1} \varsigma^{\mu}+k^{2 \mu} \varsigma\right)+k^{\mu+1}} d \varsigma\right\}\right. \\
& \left.\times\left(\|P\|_{r}-\lambda m\right)-|R| P^{\prime}(0)\left|-R^{n-1}\right| Q^{\prime}(0)||\left(\frac{\rho^{n}-\sigma^{n}}{n}-\frac{\rho^{n-2}-\sigma^{n-2}}{n-2}\right)\right],
\end{aligned}
$$

where here and throughout $Q(z)=z^{n} \overline{P(1 / \bar{z})}$.

Remark 2.1. To show that Theorem (2.3) is, in general, an improvement and generalisation of Theorem (1.1), we first prove that

$$
\begin{aligned}
& \frac{\left\{\left(\frac{\rho^{\mu}-\sigma^{\mu}}{\rho^{n}-\sigma^{n}}\right) \frac{\left|a_{\mu}\right|}{\left|a_{0}\right|-\lambda m} k^{\mu+1} R^{\mu}+R^{\mu+1}\right\}}{R^{\mu+1}+k^{\mu+1}+\left(\frac{\rho^{\mu}-\sigma^{\mu}}{\rho^{n}-\sigma^{n}}\right) \frac{\left|a_{\mu}\right|}{\left|a_{0}\right|-\lambda m}\left(k^{\mu+1} R^{\mu}+k^{2 \mu} R\right)} \\
& \times \exp \left\{n \int_{r}^{R} \frac{\frac{\mu}{n} \frac{\left|a_{\mu}\right|}{\left|a_{0}\right|-\lambda m} k^{\mu+1} \varsigma^{\mu-1}+\varsigma^{\mu}}{\varsigma^{\mu+1}+\frac{\mu}{n} \frac{\left|a_{\mu}\right|}{\left|a_{0}\right|-\lambda m}\left(k^{\mu+1} \varsigma^{\mu}+k^{2 \mu} \varsigma\right)+k^{\mu+1}} d \varsigma\right\} \\
& \leq \frac{R^{\mu}}{r^{\mu}+k^{\mu}}\left[\frac{R^{\mu}+k^{\mu}}{r^{\mu}+k^{\mu}}\right]^{\frac{n}{\mu}-1} .
\end{aligned}
$$

Since, we have that

$$
\frac{\rho^{\mu}-\sigma^{\mu}}{\rho^{n}-\sigma^{n}} \leq \frac{\mu}{n}
$$

holds for all $\rho>\sigma \geq 1$ and $1 \leq \mu \leq n$, by considering the first derivative test for the function $\phi(t)=n t^{\mu}-\mu t^{n}$, where $t \geq 1$.

Also, it is easy to see that for $R \leq k$, the function

$$
S(x)=\frac{x \frac{\left|a_{\mu}\right|}{\left|a_{0}\right|-\lambda m} k^{\mu+1} R^{\mu}+R^{\mu+1}}{R^{\mu+1}+k^{\mu+1}+x \frac{\left|a_{\mu}\right|}{\left|a_{0}\right|-\lambda m}\left(k^{\mu+1} R^{\mu}+k^{2 \mu} R\right)},
$$

is a non-decreasing function of $x$, hence by using (2.11), we get

$$
\begin{gathered}
\frac{\left(\frac{\rho^{\mu}-\sigma^{\mu}}{\rho^{n}-\sigma^{n}}\right) \frac{\left|a_{\mu}\right|}{\left|a_{0}\right|-\lambda m} k^{\mu+1} R^{\mu}+R^{\mu+1}}{R^{\mu+1}+k^{\mu+1}+\left(\frac{\rho^{\mu}-\sigma^{\mu}}{\rho^{n}-\sigma^{n}}\right) \frac{\left|a_{\mu}\right|}{\left|a_{0}\right|-\lambda m}\left(k^{\mu+1} R^{\mu}+k^{2 \mu} R\right)} \\
\leq \frac{\left(\frac{\mu}{n}\right) \frac{\left|a_{\mu}\right|}{\left|a_{0}\right|-\lambda m} k^{\mu+1} R^{\mu}+R^{\mu+1}}{R^{\mu+1}+k^{\mu+1}+\left(\frac{\mu}{n}\right) \frac{\left|a_{\mu}\right|}{\left|a_{0}\right|-\lambda m}\left(k^{\mu+1} R^{\mu}+k^{2 \mu} R\right)} .
\end{gathered}
$$


Since $R \leq k$, if we put $\varsigma=R$ in (3.21) of Lemma (3.3), we have

$$
\frac{\left(\frac{\mu}{n}\right) \frac{\left|a_{\mu}\right|}{\left|a_{0}\right|-\lambda m} k^{\mu+1} R^{\mu-1}+R^{\mu}}{R^{\mu+1}+k^{\mu+1}+\left(\frac{\mu}{n}\right) \frac{\left|a_{\mu}\right|}{\left|a_{0}\right|-\lambda m}\left(k^{\mu+1} R^{\mu}+k^{2 \mu} R\right)} \leq \frac{R^{\mu-1}}{R^{\mu}+k^{\mu}} .
$$

Combining (2.12) and (2.13), we get

$$
\frac{\left(\frac{\rho^{\mu}-\sigma^{\mu}}{\rho^{n}-\sigma^{n}}\right) \frac{\left|a_{\mu}\right|}{\left|a_{0}\right|-\lambda m} k^{\mu+1} R^{\mu}+R^{\mu+1}}{R^{\mu+1}+k^{\mu+1}+\left(\frac{\rho^{\mu}-\sigma^{\mu}}{\rho^{n}-\sigma^{n}}\right) \frac{\left|a_{\mu}\right|}{\left|a_{0}\right|-\lambda m}\left(k^{\mu+1} R^{\mu}+k^{2 \mu} R\right)} \leq \frac{R^{\mu}}{R^{\mu}+k^{\mu}},
$$

and Lemma (3.3) gives

$$
\exp \left\{n \int_{r}^{R} \frac{\frac{\mu}{n} \frac{\left|a_{\mu}\right|}{\left|a_{0}\right|-\lambda m} k^{\mu+1} \varsigma^{\mu-1}+\varsigma^{\mu}}{\varsigma^{\mu+1}+\frac{\mu}{n} \frac{\left|a_{\mu}\right|}{\left|a_{0}\right|-\lambda m}\left(k^{\mu+1} \varsigma^{\mu}+k^{2 \mu} \varsigma\right)+k^{\mu+1}} d \varsigma\right\} \leq\left(\frac{R^{\mu}+k^{\mu}}{r^{\mu}+k^{\mu}}\right)^{\frac{n}{\mu}} .
$$

On combining inequalities (2.14) and (2.15), we get (2.10). The following generalisation and refinement of Theorem (1.1) is obtained by using (2.10) in Theorem (2.3).

Theorem 2.4. If $P \in P_{n, \mu}$ and $P(z) \neq 0$ in $|z|<k, k>0$, then for every $\rho>\sigma \geq 1,0<r \leq$ $R \leq k, 0 \leq \lambda \leq 1$ and $n>2$,

$$
\begin{aligned}
\|P(\rho z)-P(\sigma z)\|_{R} \leq & \frac{R^{\mu}\left(\rho^{n}-\sigma^{n}\right)}{r^{\mu}+k^{\mu}}\left(\frac{R^{\mu}+k^{\mu}}{r^{\mu}+k^{\mu}}\right)^{\frac{n}{\mu}-1}\left\{\|P\|_{r}-m\right\} \\
& -\frac{R^{\mu}|R| P^{\prime}(0)\left|-R^{n-1}\right| Q^{\prime}(0)||}{R^{\mu}+k^{\mu}}\left(\frac{\rho^{n}-\sigma^{n}}{n}-\frac{\rho^{n-2}-\sigma^{n-2}}{n-2}\right) .
\end{aligned}
$$

Since for $\rho>\sigma \geq 1, \frac{\rho^{x}-\sigma^{x}}{x}$ is increasing in $x>0$, the expression

$$
\frac{R^{\mu}}{R^{\mu}+k^{\mu}}|R| P^{\prime}(0)\left|-R^{n-1}\right| Q^{\prime}(0)||\left(\frac{\rho^{n}-\sigma^{n}}{n}-\frac{\rho^{n-2}-\sigma^{n-2}}{n-2}\right)
$$

is non-negative. Thus for polynomials of degree $n>2$, Theorem (2.4) generalises and sharpens the bound obtained in Theorem (1.1). It is easy to see that for $\sigma=1$, the R.H.S. of (2.9) is less than or equal to the R.H.S. of (1.8). Hence, for $n>2$ and $\sigma=1$, Theorem (2.3) provides a refinement of Theorem (1.2) as well.

\section{LEMMAS}

For the proof of Theorem (2.3) we need the following lemmas.

Lemma 3.1. Let $P \in P_{n, \mu}$ and $P(z)$ does not vanish in $|z|<k$, where $k \geq 1$ then for every $\rho>\sigma \geq 1,0 \leq \lambda \leq 1, n>2$ and $|z|=1$,

$$
\begin{aligned}
|P(\rho z)-P(\sigma z)| \leq & \left(\frac{\rho^{n}-\sigma^{n}}{1+\psi_{1}(\rho)}\right)\{\|P\|-\lambda m\} \\
& -\frac{|| P^{\prime}(0)|-| Q^{\prime}(0)||}{1+\psi_{1}(\rho)}\left(\frac{\rho^{n}-\sigma^{n}}{n}-\frac{\rho^{n-2}-\sigma^{n-2}}{n-2}\right),
\end{aligned}
$$


where

$$
\psi_{1}(\rho)=k^{\mu+1}\left\{\frac{\left(\frac{\rho^{\mu}-\sigma^{\mu}}{\rho^{n}-\sigma^{n}}\right) \frac{\left|a_{\mu}\right| k^{\mu-1}}{\left|a_{0}\right|-\lambda m}+1}{\left(\frac{\rho^{\mu}-\sigma^{\mu}}{\rho^{n}-\sigma^{n}}\right) \frac{\left|a_{\mu}\right| k^{\mu+1}}{\left|a_{0}\right|-\lambda m}+1}\right\} .
$$

The above Lemma is due to Mir, Imtiaz and Dawood [14].

Lemma 3.2. If $P \in P_{n, \mu}$ and $P(z) \neq 0$ in $|z|<k, k \geq 1$, then for $0 \leq \lambda \leq 1$,

$$
\frac{\left|a_{\mu}\right| k^{\mu}}{\left|a_{0}\right|-\lambda m} \leq \frac{n}{\mu}
$$

The above result is due to Mir and Dar [[12], inequality (2.6)].

Lemma 3.3. If $P \in P_{n, \mu}$ and $P(z) \neq 0$ in $|z|<k, k>0$, then for $0<r \leq R \leq k$ and $0 \leq \lambda \leq 1$

$$
\exp \left\{n \int_{r}^{R} \frac{\frac{\mu}{n} \frac{\left|a_{\mu}\right|}{\left|a_{0}\right|-\lambda m} k^{\mu+1} \varsigma^{\mu-1}+\varsigma^{\mu}}{\varsigma^{\mu+1}+\frac{\mu}{n} \frac{\left|a_{\mu}\right|}{\left|a_{0}\right|-\lambda m}\left(k^{\mu+1} \varsigma^{\mu}+k^{2 \mu} \varsigma\right)+k^{\mu+1}} d \varsigma\right\} \leq\left(\frac{R^{\mu}+k^{\mu}}{r^{\mu}+k^{\mu}}\right)^{\frac{n}{\mu}} .
$$

Proof. The above Lemma is due to Mir and Dar [12], however for the sake of completeness we give the brief outlines of its proof. Since $P(z) \neq 0$ in $|z|<k, k>0$, the polynomial $T(z)=P(\varsigma z) \neq 0$ in $|z|<\frac{k}{\varsigma}, \frac{k}{\varsigma} \geq 1$, where $0<\varsigma \leq k$. Hence applying inequality (3.18) of Lemma (3.2) to $T(z)$, we get

$$
\frac{\left|a_{\mu}\right| \varsigma^{\mu}}{\left|a_{0}\right|-\lambda m}\left(\frac{k}{\varsigma}\right)^{\mu} \leq \frac{n}{\mu}
$$

where $m=\min _{|z|=k / \varsigma}|T(z)|=\min _{|z|=k / \varsigma}|P(\varsigma z)|=\min _{|z|=k}|P(z)|$.

Now inequality (3.20) becomes

$$
\left(\frac{\mu}{n}\right) \frac{\left|a_{\mu}\right| k^{\mu}}{\left|a_{0}\right|-\lambda m} \leq 1
$$

which is equivalent to

$$
\frac{\left(\frac{\mu}{n}\right) \frac{\left|a_{\mu}\right|}{\left|a_{0}\right|-\lambda m} k^{\mu+1} \varsigma^{\mu-1}+\varsigma^{\mu}}{\varsigma^{\mu+1}+\left(\frac{\mu}{n}\right) \frac{\left|a_{\mu}\right|}{\left|a_{0}\right|-\lambda m}\left(k^{\mu+1} \varsigma^{\mu}+k^{2 \mu} \varsigma\right)+k^{\mu+1}} \leq \frac{\varsigma^{\mu-1}}{\varsigma^{\mu}+k^{\mu}} .
$$

Integrating both sides of (3.21) with respect to $\varsigma$ from $r$ to $R$, where $0<r \leq R \leq k$, we get

$$
n \int_{r}^{R} \frac{\left(\frac{\mu}{n}\right) \frac{\left|a_{\mu}\right|}{\left|a_{0}\right|-\lambda m} k^{\mu+1} \varsigma^{\mu-1}+\varsigma^{\mu}}{\varsigma^{\mu+1}+\left(\frac{\mu}{n}\right) \frac{\left|a_{\mu}\right|}{\left|a_{0}\right|-\lambda m}\left(k^{\mu+1} \varsigma^{\mu}+k^{2 \mu} \varsigma\right)+k^{\mu+1}} d \varsigma \leq n \int_{r}^{R} \frac{\varsigma^{\mu-1}}{\varsigma^{\mu}+k^{\mu}} d \varsigma,
$$

which is equivalent to

$$
\exp \left\{n \int_{r}^{R} \frac{\left(\frac{\mu}{n}\right) \frac{\left|a_{\mu}\right|}{\left|a_{0}\right|-\lambda m} k^{\mu+1} \varsigma^{\mu-1}+\varsigma^{\mu}}{\varsigma^{\mu+1}+\left(\frac{\mu}{n}\right) \frac{\left|a_{\mu}\right|}{\left|a_{0}\right|-\lambda m}\left(k^{\mu+1} \varsigma^{\mu}+k^{2 \mu} \varsigma\right)+k^{\mu+1}} d \varsigma\right\} \leq\left(\frac{k^{\mu}+R^{\mu}}{k^{\mu}+r^{\mu}}\right)^{\frac{n}{\mu}},
$$

which proves Lemma (3.3) completely. 
Lemma 3.4. If $P \in P_{n, \mu}$ and $P(z) \neq 0$ in $|z|<k, k>0$, then for $0<r \leq R \leq k$ and $0 \leq \lambda \leq 1$

$$
\begin{aligned}
\|P\|_{r} & \geq \exp \left\{-n \int_{r}^{R} \frac{\left(\frac{\mu}{n}\right) \frac{\left|a_{\mu}\right|}{\left|a_{0}\right|-\lambda m} k^{\mu+1} t^{\mu-1}+t^{\mu}}{t^{\mu+1}+k^{\mu+1}+\left(\frac{\mu}{n}\right) \frac{\left|a_{\mu}\right|}{\left|a_{0}\right|-\lambda m}\left(k^{\mu+1} t^{\mu}+k^{2 \mu} t\right)} d t\right\}\|P\|_{R} \\
& +\left[1-\exp \left\{-n \int_{r}^{R} \frac{\left(\frac{\mu}{n}\right) \frac{\left|a_{\mu}\right|}{\left|a_{0}\right|-\lambda m} k^{\mu+1} t^{\mu-1}+t^{\mu}}{t^{\mu+1}+k^{\mu+1}+\left(\frac{\mu}{n}\right) \frac{\left|a_{\mu}\right|}{\left|a_{0}\right|-\lambda m}\left(k^{\mu+1} t^{\mu}+k^{2 \mu} t\right)} d t\right\}\right] m .
\end{aligned}
$$

The above result is due to Mir and Dar [[12], Corollary 1].

\section{Proof of THE THEOREM}

Proof of Theorem 2.3. Since $P \in P_{n, \mu}$ and $P(z) \neq 0$ in $|z|<k, k>0$, the polynomial $F(z)=P(R z)$ has no zeros in $|z|<k / R, k / R \geq 1$. Now applying inequality (3.17) of Lemma 3.1 to the polynomial $F(z)$, we have for every $\rho>\sigma \geq 1$ and $n>2$,

$$
\begin{aligned}
& \|F(\rho z)-F(\sigma z)\| \leq \frac{1}{1+(k / R)^{\mu+1}\left\{\frac{\left(\frac{\rho^{\mu}-\sigma^{\mu}}{\rho^{n}-\sigma^{n}}\right) \frac{\left|a_{\mu}\right|}{\left|a_{0}\right|-\lambda m} R^{\mu}(k / R)^{\mu-1}+1}{\left(\frac{\rho^{\mu}-\sigma^{\mu}}{\rho^{n}-\sigma^{n}}\right) \frac{\left|a_{\mu}\right|}{\left|a_{0}\right|-\lambda m} R^{\mu}(k / R)^{\mu+1}+1}\right\}} \\
& \times\left[\left(\rho^{n}-\sigma^{n}\right)(\|F\|-\lambda m)-|| F^{\prime}(0)|-| H^{\prime}(0)||\left(\frac{\rho^{n}-\sigma^{n}}{n}-\frac{\rho^{n-2}-\sigma^{n-2}}{n-2}\right)\right],
\end{aligned}
$$

where $m=\min _{|z|=k / R}|F(z)|=\min _{|z|=k / R}|P(R z)|=\min _{|z|=k}|P(z)|$ and $H(z)=z^{n} \overline{F(1 / \bar{z})}$.

This gives

$$
\begin{gathered}
\|P(R \rho z)-P(R \sigma z)\| \leq \frac{\left\{\left(\frac{\rho^{\mu}-\sigma^{\mu}}{\rho^{n}-\sigma^{n}}\right) \frac{\left|a_{\mu}\right|}{\left|a_{0}\right|-\lambda m} k^{\mu+1} R^{\mu}+R^{\mu+1}\right\}}{R^{\mu+1}+k^{\mu+1}+\left(\frac{\rho^{\mu}-\sigma^{\mu}}{\rho^{n}-\sigma^{n}}\right) \frac{\left|a_{\mu}\right|}{\left|a_{0}\right|-\lambda m}\left(k^{\mu+1} R^{\mu}+k^{2 \mu} R\right)} \\
\times\left[\left(\rho^{n}-\sigma^{n}\right)\left(\|P\|_{R}-m\right)-|R| P^{\prime}(0)\left|-R^{n-1}\right| Q^{\prime}(0)||\left(\frac{\rho^{n}-\sigma^{n}}{n}-\frac{\rho^{n-2}-\sigma^{n-2}}{n-2}\right)\right],
\end{gathered}
$$

for every $\rho>\sigma \geq 1$ and $0<R \leq k$.

Now if $0<r \leq R \leq k$, then by using (3.22) of Lemma 3.4 in (4.23), we obtain

$$
\begin{aligned}
& \|P(\rho z)-P(\sigma z)\|_{R} \leq \frac{\left\{\left(\frac{\rho^{\mu}-\sigma^{\mu}}{\rho^{n}-\sigma^{n}}\right) \frac{\left|a_{\mu}\right|}{\left|a_{0}\right|-\lambda m} k^{\mu+1} R^{\mu}+R^{\mu+1}\right\}}{R^{\mu+1}+k^{\mu+1}+\left(\frac{\rho^{\mu}-\sigma^{\mu}}{\rho^{n}-\sigma^{n}}\right) \frac{\left|a_{\mu}\right|}{\left|a_{0}\right|-\lambda m}\left(k^{\mu+1} R^{\mu}+k^{2 \mu} R\right)} \\
& \times\left[\left(\rho^{n}-\sigma^{n}\right) \exp \left\{n \int_{r}^{R} \frac{\left(\frac{\mu}{n}\right) \frac{\left|a_{\mu}\right|}{\left|a_{0}\right|-\lambda m} k^{\mu+1} \varsigma^{\mu}+\varsigma^{\mu+1}}{\varsigma^{\mu+1}+k^{\mu+1}+\left(\frac{\mu}{n}\right) \frac{\left|a_{\mu}\right|}{\left|a_{0}\right|-\lambda m}\left(k^{\mu+1} \varsigma^{\mu}+k^{2 \mu} \varsigma\right)} d \varsigma\right\}\right. \\
& \left.\times\left(\|P\|_{r}-m\right)-|R| P^{\prime}(0)\left|-R^{n-1}\right| Q^{\prime}(0) \mid\left(\frac{\rho^{n}-\sigma^{n}}{n}-\frac{\rho^{n-2}-\sigma^{n-2}}{n-2}\right)\right],
\end{aligned}
$$

which is (2.9) and this completes the proof of Theorem (2.3). 
Acknowledgements. The work is sponsored by UGC, Govt. of India under the Major Research Project Scheme vide no. MRP-MAJOR-MATH-2013-29143.

The authors are very grateful to the referees for their valuable suggestions regarding the paper.

\section{REFERENCES}

[1] Aziz, A. and Aliya, Q.,Growth of polynomials not vanishing in a disk of prescribed radius, Int. J. Pure Appl. Math., 41 (2007), 713-735

[2] Aziz, A. and Shah, W. M., Inequalities for a polynomial and its derivative, Math. Ineq. Appl., 7 (2004), 379-391

[3] Bidkham, M. and Dewan, K. K., Inequalities for a polynomial and its derivative, J. Math. Anal. Appl., 166 (1992), 319-324

[4] Chanam, B. and Dewan, K. K., Inequalities for a polynomial and its derivative, J. Math. Anal. Appl., 336 (2007), 171-179

[5] Dewan, K.K., Singh, N. and Mir, A., Extensions of some polynomial inequalities to the polar derivative, J. Math. Anal. Appl., 352 (2009), 807-815

[6] Gardner, R. B., Govil, N. K. and Weems, A., Growth of polynomials not vanishing inside a circle, Int. J. Pure Appl. Math., 13 (2004), 491-498

[7] Gardner, R. B., Govil, N. K. and Weems, A., Some results concerning rate of growth of polynomials, East J. Approx., 10 (2004), 301-312

[8] Gardner, R. B., Govil, N. K. and Musukula, S. R., Rate of growth of polynomials not vanishing inside a circle, J. Ineq. Pure Appl. Math., 6 (2005),(Issue 2, Art. 53): 1-9

[9] Govil, N. K. and Nyuydinkong, G. N., On the maximum modulus of polynomials not vanishing inside a circle, J. Interdisciplinary Math., 4 (2001), 93-100

[10] Mir, A., Dewan, K. K. and Singh, N., Some inequalities concerning the rate of growth of polynomials, Turk. J. Math., 33 (2009), 239-247

[11] Mir, A., Dewan, K. K. and Singh, N., Some $L^{p}$ inequalities for polynomials, Funct. Approx. Comment. Math., 42 (2010), part 2, 131-143

[12] Mir, A. and Dar, B., Inequalities concerning the rate of growth of polynomials, Afrika Mate., 27 (2016), 279-290

[13] Mir, A., Dewan, K. K., Singh, N. and Dar, B., Some integral inequalities for polynomials with restricted zeros, Southeast Asian Bull. Math., 38 (2014),83-92

[14] Mir, A., Hussain, I. and Dawood, Q. M., Some inequalities concerning rate of growth of polynomials, Anal. Theory Appl., 30 (2014), 290-295

[15] Rahman, Q. I. and Schmeisser, G., Analytic theory of polynomials, Oxford University Press Inc. New York, 2002

DEPARTMENT OF MATHEMATICS

UNIVERSITY OF KASHMIR

HAZRATBAL, 190006, SRINAGAR, INDIA

Email address: mabdullah_mir@yahoo.co.in

Email address: qdawoodegmail .com 